\title{
Music and Mood Regulation during the early-stages of the COVID-19 Pandemic
}

\author{
Sarah Hennessy ${ }^{\mathbb{1}}$, Matthew Sachs ${ }^{\mathbb{1} 2}$, Jonas Kaplan ${ }^{1}$, \& Assal Habibi ${ }^{* 1}$ \\ ${ }^{1}$ Brain and Creativity Institute, University of Southern California, Los Angeles, CA, United States \\ ${ }^{2}$ Center for Science and Society, Columbia University, New York, NY, United States \\ "Co-first authors, these authors contributed equally to this work \\ *corresponding author, ahabibi@usc.edu
}

\begin{abstract}
Music-listening can be an effective strategy for regulating affect, leading to positive wellbeing. However, it is unclear how differences in culture and disposition can impact music's affective benefits. The COVID-19 pandemic provides a unique opportunity to study how music is used to cope with stress, loss, and unease across the world. We used an online survey to test if people from four different countries used music to manage their emotions during quarantine and if the functions of music depended on empathy, anxiety, depression, or country of residence. We found a positive relationship between the use of music-listening for affect regulation and current wellbeing, particularly for participants from India. While people with stronger symptoms of depression and anxiety used music differently, the end result was still a positive change in affect. Our findings highlight the universality of music's affective potency and its ability to help people manage an unprecedented life stressor.
\end{abstract}

Keywords: emotion, music, COVID-19, well-being 
In March 2020, the COVID-19 outbreak forced people across the globe into quarantine. Communities around the world sought innovative ways to cope with growing anxiety, uncertainty, boredom, and social isolation. Music seemed to provide solace. Videos of apartment block performances, synchronous nightly cheering, and collective sing-a-longs from all over the world were shared widely across the internet. When Italian tenor Andrea Bocelli performed a solo Easter concert from an empty Milan Cathedral, the broadcast was viewed 35 million times.

These anecdotes suggest that across cultures, music-listening helped people cope with the stress, uncertainty, and despair that stemmed from the pandemic. Music-listening can be beneficial for health and well-being across the lifespan (Hanser \& Mandel, 2010; Laukka, 2007; Miranda \& Gaudreau, 2011; Saarikallio, 2011), and listening to music reduces self-reported feelings of anxiety, and physiological measures of stress (Panteleeva et al., 2018). Music may also alleviate symptoms related to major psychological and mental disorders (Chan et al., 2011; Loue et al., 2008) by helping people cope with negative affective states (Miranda \& Claes, 2009) and to balance their mood (Västfjäll, 2002).

One way music listening can impact wellbeing is through its ability to induce strong emotions (Habibi \& Damasio, 2014; Juslin \& Laukka, 2004; Sloboda et al., 2001). Indeed, its emotional potency is consistently cited as one of the main reasons that people, across demographic groups, listen to music (Randall \& Rickard, 2017). Because of this, music is also recognized as an effective tool for affect regulation, i.e. the process of changing the incidence, duration, and/or intensity of an affective state. We therefore hypothesized that in response to a stressful event, like the COVID-19 pandemic, people would report using music to cope precisely because of its ability to alter their emotional state.

There are several mechanisms by which music-listening can alter one's affective states: by relaxing us (Saarikallio \& Erkkilä, 2007; van Goethem \& Sloboda, 2011), by distracting us from a negative mood (Thoma et al., 2012), or by allowing us to purge negative emotions (Van den Tol \& Edwards, 2014). Recently, the Brief Music in Mood Regulation Scale (B-MMR) was created to capture these various regulatory strategies (Saarikallio, 2012). The scale, and many of its subcomponents, are associated with general emotion regulation through reappraisal and using music to reappraise leads to increased psychological well-being (Chin \& Rickard, 2012).

Interestingly, the Discharge subscale, which refers to selecting music that reflects one's current negative affective state in order to release or purge these feelings, is not positively correlated with reappraisal. This subscale is also associated with symptoms of depression and anxiety (Thomson et al., 2014; Carlson et al. 2015). However, it remains uncertain if individuals who are more anxious or depressed use angry or sad music to feel better or if listening to this music leads to stronger feelings of anxiety or depression. The impact of music on mood likely depends on the goal of the user, the situation, individual differences, the type of emotion regulation strategy being used, and their pre-existing mood (Saarikallio \& Erkkilä, 2007). One individual difference measure that may influence this relationship is trait empathy. A particular component of empathy, Fantasy, which refers to the tendency to become absorbed into the situations of characters in stories, movies, and music, has been linked to the enjoyment of negative-valenced music (Sachs et al., 2020). It is therefore possible that the degree to which listening to sad or angry music can improve mood and well-being depends on this trait. 
The COVID-19 pandemic provides a unique opportunity in which to study cross-cultural responses to a singular, unifying stressor on a grand scale. While there is evidence that, across cultures, people choose to listen to music because of its affective function, certain international differences have been identified (Schäfer et al., 2012; Boer et al., 2012). Given these findings, we expect people to universally use music to regulate their emotions, but that the strategies, effectiveness, and qualities of the music-listening may differ across cultures. Furthermore, while laboratory studies have shown that music listening can aid in autonomic recovery after induction of acute stress (de la Torre-Luque et al., 2017; Suda et al., 2008; Thoma et al., 2013) and trauma (Zoteyeva et al., 2016), there are relatively few studies on how individuals use music to regulate their emotions effectively in response to an acute stressor, such as in the context of significant, global event.

92

93

94

95

96

97

98

99

100

101

102

103

104

105

106

107

108

109

110

111

112

113

114

115

116

117

118

119

120

121

To this end, we conducted a large scale multi-national study with a diverse population to explore the role of music listening in mood and emotion regulation during the COVID-19 pandemic. Through on-line surveys we aimed to investigate how people across the globe use music to regulate their mood and alleviate the discomfort and sadness of social isolation. There were four main hypotheses. One, that across cultures, individuals more personally affected by COVID-19 would report using music to regulate their mood. Two, that individual differences in trait measures of empathy as well as symptoms of depression and anxiety would be associated with different regulatory strategies with music. Three, that the relationship between music-listening emotion regulation strategies and improvements in mood and wellbeing would be moderated by these trait differences. Four, that these differences would be reflected in the songs that people chose to listen to during the pandemic.

\section{Methods}

\section{Participants}

An online survey was distributed to individuals currently living in Italy, the United Kingdom and the United States, through Prolific.co (Palan \& Schitter, 2018) on April 6th, 2020 and April 7th, 2020. After removing participants for improper responses (i.e. missing the attention check or responding too quickly), this left 148 participants from the United States (Mage $=29.16,86$ female), 136 from Italy $\left(M_{\text {age }}=25.81,59\right.$ female $)$, and 150 from the UK $\left(M_{\text {age }}=\right.$ 35.83, 89 female). Participants currently living in India $\left(N=155, M_{a g e}=33.47,57\right.$ female) were recruited through using CloudResearch's Prime Panels, an online platform with more than 50 million participants across the globe, between April 24th and April 27th. On both platforms, additional inclusion criteria included being a citizen of the country in which one resides, being a native English speaker, and being between the ages of 18-65. In total, 589 people completed the survey. For COVID-19 case rates at the time of data collection, see Table 1.

\begin{tabular}{|c|c|c|c|c|c|c|}
\hline & & Total & India & Italy & $\begin{array}{l}\text { United } \\
\text { States }\end{array}$ & $\begin{array}{l}\text { United } \\
\text { Kingdom }\end{array}$ \\
\hline \multicolumn{7}{|l|}{ Gender } \\
\hline & $n$ & 589 & 155 & 136 & 148 & 150 \\
\hline & $\%$ Female & 49 & 37 & 43 & 58 & 59 \\
\hline \multicolumn{7}{|l|}{ Age } \\
\hline & Mean & 31.22 & 33.11 & 25.81 & 29.16 & 35.83 \\
\hline
\end{tabular}


10.29

SD
Cases/100k
Deaths/100k

10.6

6.05

9.15

11.38

COVID-19

Table 1. Demographic and COVID-19 characteristics for study sample by country. COVID-19 data reflects cases per 100,000 on the day the survey was distributed in each country, as reported by Johns Hopkins Coronavirus Resource Center.

\section{Survey materials}

The survey included several previously-published questionnaires designed to assess a variety of individual difference measures. These questionnaires and a description of each are listed below:

A. Patient Health Questionnaire (PHQ; Kroenke et al., 2001): a brief measure of symptoms/severity of depression

B. State and Trait Anxiety Index (STAI; Spielberger, 2010): a state and trait anxiety measure

C. Emotion Regulation Quotient (ERQ; Gross \& John, 2003): a widely used measure of two types of emotional regulation strategies, cognitive reappraisal and expressive suppression

D. Interpersonal Reactivity Index (IRI; Davis, 1980): a measure of empathy that assesses four subcomponents, including Fantasy, Perspective Taking, Empathic Concern, and Personal Distress

E. Brief Music and Mood Questionnaire (Saarikallio, 2012): a 21-item self-report instrument for assessing the use of seven different music-related moodregulation strategies. These include
a. Entertainment
b. Revival
c. Strong sensation
d. Diversion
e. Discharge
f. Mental work
g. Solace

In addition to these questionnaires, participants were asked about their music listening habits, both currently and a year ago (prior to the onset of the COVID-19 pandemic). Specifically participants were asked how many hours did they listen to music on an average day/a year ago and how many hours are they currently listening to music, what genre of music they most commonly listen to currently/listened to a year ago, and to list 5 songs that they are frequently listening to/listened to a year ago. While we understand that asking participants to retrospectively report their music listening habits from a year ago may be unreliable and subject to certain biases, we wanted to control for the fact that music preferences might change given the time of year for example during the holidays. Therefore, in order to directly compare changes before and after the COVID-19 pandemic, we felt that it was best to ask about listening habits during the same time of year, a year prior. After listing songs that they are currently listening to, we directly asked participants the degree to which listening to music had made 
them feel better during the pandemic by asking how strongly they agree/disagree with the following statements:

1. I have been turning to music listening or playing to take my mind off things

2. I find music listening or playing to be helpful in coping with the current crisis.

3. I have been listening to music more than usual to make myself feel better.

Participants additionally answered several YES/NO questions to assess distress, perceived risk, and change of mood related to the COVID-19 pandemic (see Supplementary Materials for details).

Finally, demographic questions were collected including a brief measure of socioeconomic status (using the MacArthur Scale of Subjective Social Status; Adler et al., 2000), years of musical training, age, and gender.

\section{Statistical analyses}

Statistical analyses were performed using $R$ (R Core Team, 2020). For all tests for significance, an alpha level of 0.05 was used. To assess the degree to which each individual was personally affected by the COVID-19 pandemic, a continuous measure of COVID severity was calculated by totaling the number of YES responses for each participant to the four COVIDrelated questions in the survey (did you personally test positive, were you personally hospitalized, did you experience financial loss, are there shelter-in-place orders where you are) and adding this to the question regarding degree of self-isolating ( $4-100 \%, 1$ - none). This measure of objective hardship related to COVID-19 will subsequently be referred to as COVID severity. In addition to our stated hypotheses, as a sanity-check, we tested for differences between countries in COVID-19 perceived risk and severity, as well as scores on the PHQ-9, STAI, and ERQ, while controlling for age, gender, education, and SES using ANCOVA models. For all ANOVA models, post-hoc comparisons between countries were conducted using Tukey's Honest Significant Difference.

\section{Music in mood regulation by country and COVID-19 severity.}

Per our first hypothesis, we tested whether differences exist across countries in terms of the ways in which music is used to regulate mood. To this end, a series of separate 1-way ANCOVAs were conducted to assess the effects of country and COVID-19 personal risk on subscales, and overall scores, on the B-MMR, while controlling for age, gender, education, SES, and musicianship.

\section{Individual differences and the function of music to regulate mood.}

To test our second hypothesis, separate 1-way ANCOVAs were conducted to assess the effects of state anxiety (STAI), depressive symptoms (PHQ-9), and empathy (IRI) on B-MMR scores and subscales, while controlling for country, age, gender, education, SES, and musicianship.

\section{Using music to regulate mood and wellbeing.}

Per our third hypothesis, we assessed the degree to which music-listening improved mood, as well as the interaction between personality, music regulatory strategies and improvements in mood.

\section{Changes in music listening habits.}

To assess changes in music listening habits and preferences during the COVID-19 pandemic, Spotify playlists were created that contained the songs that participants reported 
listening to pre-COVID and during COVID. Playlists were analyzed using a Python library to access data on Spotify's Web API https://github.com/plamere/spotipy. Auditory features were extracted from each song, which included:

1. Acousticness: real value between 0 and 1 measuring the likeliness that the track is acoustic

2. Danceability: real value between 0 and 1 measuring how danceable the song is

3. Energy: real value between 0 and 1 measuring intensity and activity;

4. Valence: real value between 0 and 1 measuring whether the song inspires positive/negative emotions

5. Tempo: real value expressed in beats per minute.

6. Loudness: real value between -60 and 0 measuring how loud the song is

7. Mode: integer ( 0 or 1 ) indicates the modality (major or minor) of a track, the type of scale from which its melodic content is derived (major is represented by 1 and minor is 0 )

Separate repeated-measures ANOVAs were conducted on each acoustic variable, with country as the between-subjects factor, and time (pre- or post-COVID) as the within-subjects factor. Additional analyses on the music selected pre and post COVID are included in the supplementary materials.

\section{Results}

\section{Responses to COVID-19 by country. \\ COVID-19 severity and perceived risk.}

A significant effect of country was observed in COVID-19 severity $(F(3,545)=19.67$, $p$ $<0.0001, \eta^{2}=0.09$ ), where participants from Italy reported higher severity than those from the United Kingdom $(p<0.0001)$ and the United States $(p<0.0001)$ (see Figure 1). Participants from India reported higher severity than those from the United Kingdom $(p<0.001)$, and the United States $(p<0.0001)$. No differences were observed between countries in perceived personal risk of COVID-19 ( $p>0.05)$.
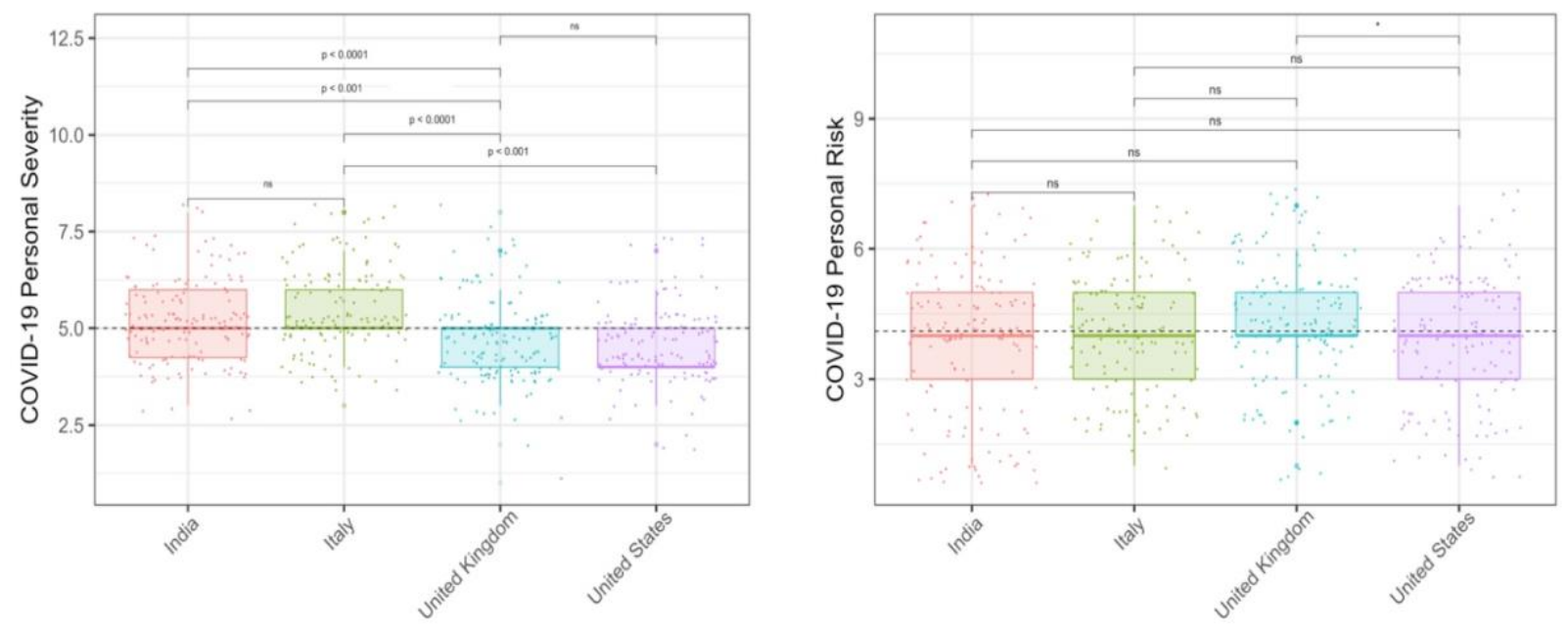

Fig 1. Differences between countries in personal COVID-19 severity and perceived risk. 
No differences were observed between countries in symptoms of depression (PHQ-9; $\mathrm{p}$ $>0.05)$. There was a significant effect of country on state anxiety $(F(3,545)=4.49, p<0.01$, $\left.\eta^{2}=0.02\right)$, where participants from Italy had greater anxiety than those from the United States $(p$ $<0.05)$. Differences between countries on trait anxiety were not significant after correcting for multiple comparisons $(p>0.05)$. Unsurprisingly, across all four countries, symptoms of both depression and anxiety were associated with increased feelings of anxiety and depression during the pandemic (anxiety: $r=-0.25, p<0.001$; depression: $r=-0.46, p<0.001$ ).

\section{Music and mood regulation by country and COVID severity.}

A significant effect of country was observed on Music and Mood Regulation total score $\left(F(3,545)=7.70, p<0.0001, \eta^{2}=0.03\right)$, where participants from India scored higher than those from Italy $(p<0.05)$, the United Kingdom $(p<0.001)$, and the United States $(p<0.01$; see Figure 2). In addition, several subscales of the B-MMR differed significantly by country. Briefly, the strategies Revival, Diversion, Solace, and Mental Work were all more likely to be endorsed by participants from India than those from Italy, the United States or the UK. Furthermore, the strategy of Sensation was more likely to be endorsed by participants from Italy and India than those from the UK or USA (see Table 2). No effect of country was observed for the Discharge nor Entertainment strategies.

275

\begin{tabular}{|c|c|c|c|c|c|c|}
\hline $\begin{array}{c}\text { Sum } \\
\text { of } \\
\text { Squares }\end{array}$ & $d f$ & $\begin{array}{l}\text { Mean } \\
\text { Square }\end{array}$ & $F$ & $p$ & partial $\eta^{2}$ & $\begin{array}{c}\text { partial } \eta^{2} \\
90 \% \mathrm{CI} \\
{[\mathrm{LL}, \mathrm{UL}]}\end{array}$ \\
\hline
\end{tabular}

MMR Discharge

\begin{tabular}{rrrrrcrl}
\hline (Intercept) & 3378.58 & 1 & 3378.58 & 316.84 & .000 & & \\
age & 259.37 & 1 & 259.37 & 24.32 & $.000^{* * * *}$ & .04 & {$[.02, .07]$} \\
education & 0.07 & 1 & 0.07 & 0.01 & .934 & .00 & {$[.00, .00]$} \\
gender & 80.46 & 1 & 80.46 & 7.55 & $.006^{* *}$ & .01 & {$[.00, .03]$} \\
SES & 48.10 & 1 & 48.10 & 4.51 & .034 & .01 & {$[.00, .03]$} \\
musician & 120.39 & 1 & 120.39 & 11.29 & $.001^{* *}$ & .02 & {$[.01, .04]$} \\
country & 74.32 & 3 & 24.77 & 2.32 & .074 & .01 & {$[.00, .03]$} \\
Error & 5811.57 & 545 & 10.66 & & & & \\
\hline
\end{tabular}

MMR Diversion

\begin{tabular}{rrrrrrrr} 
(Intercept) & 3136.86 & 1 & 3136.86 & 424.07 & .000 & & \\
age & 144.80 & 1 & 144.80 & 19.58 & $.000^{* * *}$ & .03 & {$[.01, .06]$} \\
education & 0.12 & 1 & 0.12 & 0.02 & .900 & .00 & {$[.00, .00]$} \\
gender & 5.13 & 1 & 5.13 & 0.69 & .405 & .00 & {$[.00, .01]$} \\
SES & 0.93 & 1 & 0.93 & 0.13 & .723 & .00 & {$[.00, .01]$} \\
musician & 35.36 & 1 & 35.36 & 4.78 & .029 & .01 & {$[.00, .03]$} \\
country & 220.27 & 3 & 73.42 & 9.93 & $.000^{* * *}$ & .05 & {$[.02, .08]$} \\
Error & 4031.40 & 545 & 7.40 & & & & \\
\hline
\end{tabular}


MMR Entertainment

\begin{tabular}{rrrrrrrr}
\hline (Intercept) & 3658.52 & 1 & 3658.52 & 515.85 & .000 & & \\
age & 125.64 & 1 & 125.64 & 17.72 & $.000^{* * *}$ & .03 & {$[.01, .06]$} \\
education & 0.54 & 1 & 0.54 & 0.08 & .783 & .00 & {$[.00, .01]$} \\
gender & 16.02 & 1 & 16.02 & 2.26 & .133 & .00 & {$[.00, .02]$} \\
SES & 2.14 & 1 & 2.14 & 0.30 & .583 & .00 & {$[.00, .01]$} \\
musician & 28.36 & 1 & 28.36 & 4.00 & $.046^{*}$ & .01 & {$[.00, .02]$} \\
country & 51.22 & 3 & 17.07 & 2.41 & .066 & .01 & {$[.00, .03]$} \\
Error & 3865.26 & 545 & 7.09 & & & & \\
\hline
\end{tabular}

280

281

MMR Mental Work

\begin{tabular}{rrrrrrrl}
\hline (Intercept) & 3193.43 & 1 & 3193.43 & 486.49 & .000 & & \\
age & 73.06 & 1 & 73.06 & 11.13 & $.001^{* *}$ & .02 & {$[.01, .04]$} \\
education & 2.66 & 1 & 2.66 & 0.41 & .524 & .00 & {$[.00, .01]$} \\
gender & 11.83 & 1 & 11.83 & 1.80 & .180 & .00 & {$[.00, .02]$} \\
SES & 3.99 & 1 & 3.99 & 0.61 & .436 & .00 & {$[.00, .01]$} \\
musician & 57.69 & 1 & 57.69 & 8.79 & $.003^{* * *}$ & .02 & {$[.00, .04]$} \\
country & 127.70 & 3 & 42.57 & 6.48 & $.000^{* * *}$ & .03 & {$[.01, .06]$} \\
Error & 3577.47 & 545 & 6.56 & & & & \\
\hline
\end{tabular}

282

283

MMR Revival

\begin{tabular}{rrrrrrrr}
\hline (Intercept) & 2946.89 & 1 & 2946.89 & 383.75 & .000 & & \\
age & 134.19 & 1 & 134.19 & 17.47 & $.000^{* * *}$ & .03 & {$[.01, .06]$} \\
education & 2.01 & 1 & 2.01 & 0.26 & .609 & .00 & {$[.00, .01]$} \\
gender & 5.24 & 1 & 5.24 & 0.68 & .409 & .00 & {$[.00, .01]$} \\
SES & 0.93 & 1 & 0.93 & 0.12 & .728 & .00 & {$[.00, .01]$} \\
musician & 25.51 & 1 & 25.51 & 3.32 & .069 & .01 & {$[.00, .02]$} \\
country & 197.08 & 3 & 65.69 & 8.55 & $.000^{* * *}$ & .04 & {$[.02, .07]$} \\
Error & 4185.20 & 545 & 7.68 & & & & \\
\hline
\end{tabular}

284

285

MMR Sensation

\begin{tabular}{rrrrrrrl}
\hline (Intercept) & 3431.10 & 1 & 3431.10 & 570.04 & .000 & & \\
age & 46.23 & 1 & 46.23 & 7.68 & $.006^{* *}$ & .01 & {$[.00, .03]$} \\
education & 1.88 & 1 & 1.88 & 0.31 & .577 & .00 & {$[.00, .01]$} \\
gender & 4.99 & 1 & 4.99 & 0.83 & .363 & .00 & {$[.00, .01]$} \\
SES & 1.11 & 1 & 1.11 & 0.18 & .668 & .00 & {$[.00, .01]$} \\
musician & 110.66 & 1 & 110.66 & 18.38 & $.000^{* * *}$ & .03 & {$[.01, .06]$} \\
country & 127.01 & 3 & 42.34 & 7.03 & $.000^{* * *}$ & .04 & {$[.01, .06]$} \\
Error & 3280.38 & 545 & 6.02 & & & &
\end{tabular}

286

287 MMR Solace

\begin{tabular}{rrrrrrrr}
\hline (Intercept) & 3342.79 & 1 & 3342.79 & 481.94 & .000 & & \\
age & 66.31 & 1 & 66.31 & 9.56 & $.002^{* * *}$ & .02 & {$[.00, .04]$} \\
education & 4.68 & 1 & 4.68 & 0.67 & .412 & .00 & {$[.00, .01]$}
\end{tabular}




$\begin{array}{rrrrrrrr}\text { gender } & 19.01 & 1 & 19.01 & 2.74 & .098 & .01 & {[.00, .02]} \\ \text { SES } & 8.06 & 1 & 8.06 & 1.16 & .282 & .00 & {[.00, .01]} \\ \text { musician } & 33.60 & 1 & 33.60 & 4.84 & .028^{*} & .01 & {[.00, .03]} \\ \text { country } & 168.66 & 3 & 56.22 & 8.11 & .000^{* * *} & .04 & {[.02, .07]} \\ \text { Error } & 3780.14 & 545 & 6.94 & & & & \end{array}$

Note. LL and UL represent the lower-limit and upper-limit of the partial $\eta^{2}$ confidence interval, respectively.

Table 2. ANCOVA results, country predicting each Brief Music and Mood Regulation strategy.

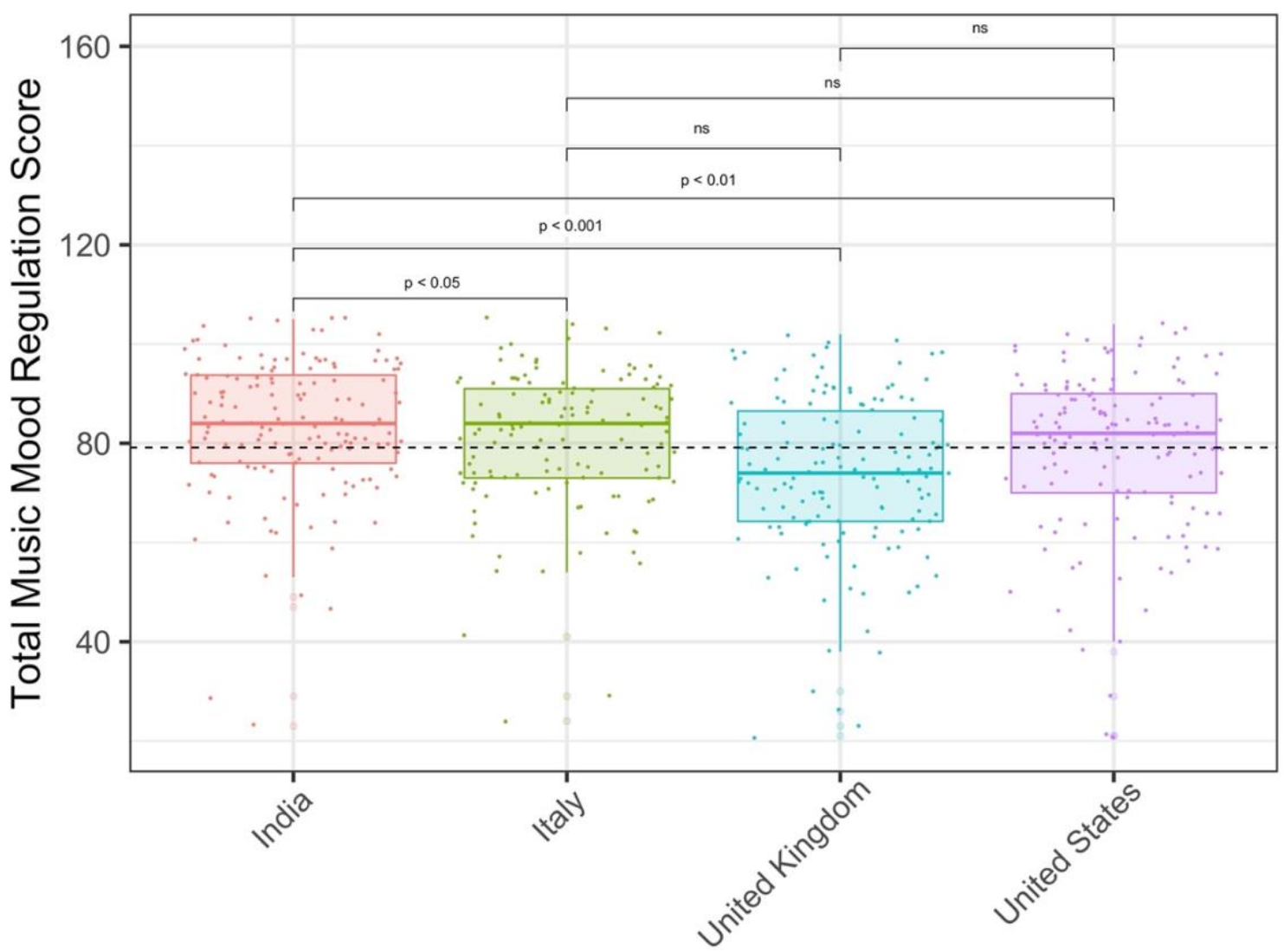

Fig 2. Differences between countries in total music and mood regulation score (B-MMR Total Score)

A significant effect of personal COVID-19 risk was observed on music and mood regulation total score, across countries, $\left(F(1,547)=9.61, p<0.05, \eta^{2}=0.02\right)$, where personal risk of COVID-19 was positively correlated with use of music for mood regulation ( $\boldsymbol{\beta}=0.13$; see Figure 3 ) as well as with the Diversion subscale $\left(F(1,547)=15.77, \boldsymbol{\beta}=0.17, p<0.0001, \eta^{2}=0.03\right)$. No effects of personal COVID-19 risk were observed for any other B-MMR strategy. Additionally, a significant effect of COVID-19 severity was observed on music and mood regulation total score, across 
countries $\left(F(1,547)=13.17, p<0.001, \eta^{2}=0.02\right)$, where COVID-19 severity was positively correlated with total music mood regulation score $(\boldsymbol{\beta}=0.15)$. The Diversion $(F(1,547)=5.70, \boldsymbol{\beta}$ $\left.=0.10, p<0.05, \eta^{2}=0.01\right)$, Entertainment $\left(F(1,547)=5.25, \boldsymbol{\beta}=0.10, p<0.05, \eta^{2}=0.00\right)$, Revival $\left(F(1,547)=11.27, \boldsymbol{\beta}=0.15, p<0.001, \eta^{2}=0.02\right)$, Sensation $(F(1,547)=15.16, \boldsymbol{\beta}=$ $\left.0.16, p<0.001, \eta^{2}=0.02\right)$, Solace $\left(F(1,547)=12.22, \beta=0.15, p<0.001, \eta^{2}=0.02\right)$, and Mental Work $\left(F(1,547)=13.75, \boldsymbol{\beta}=0.16, p<0.001, \eta^{2}=0.02\right)$, subscales were also predicted by COVID-19 severity across countries.

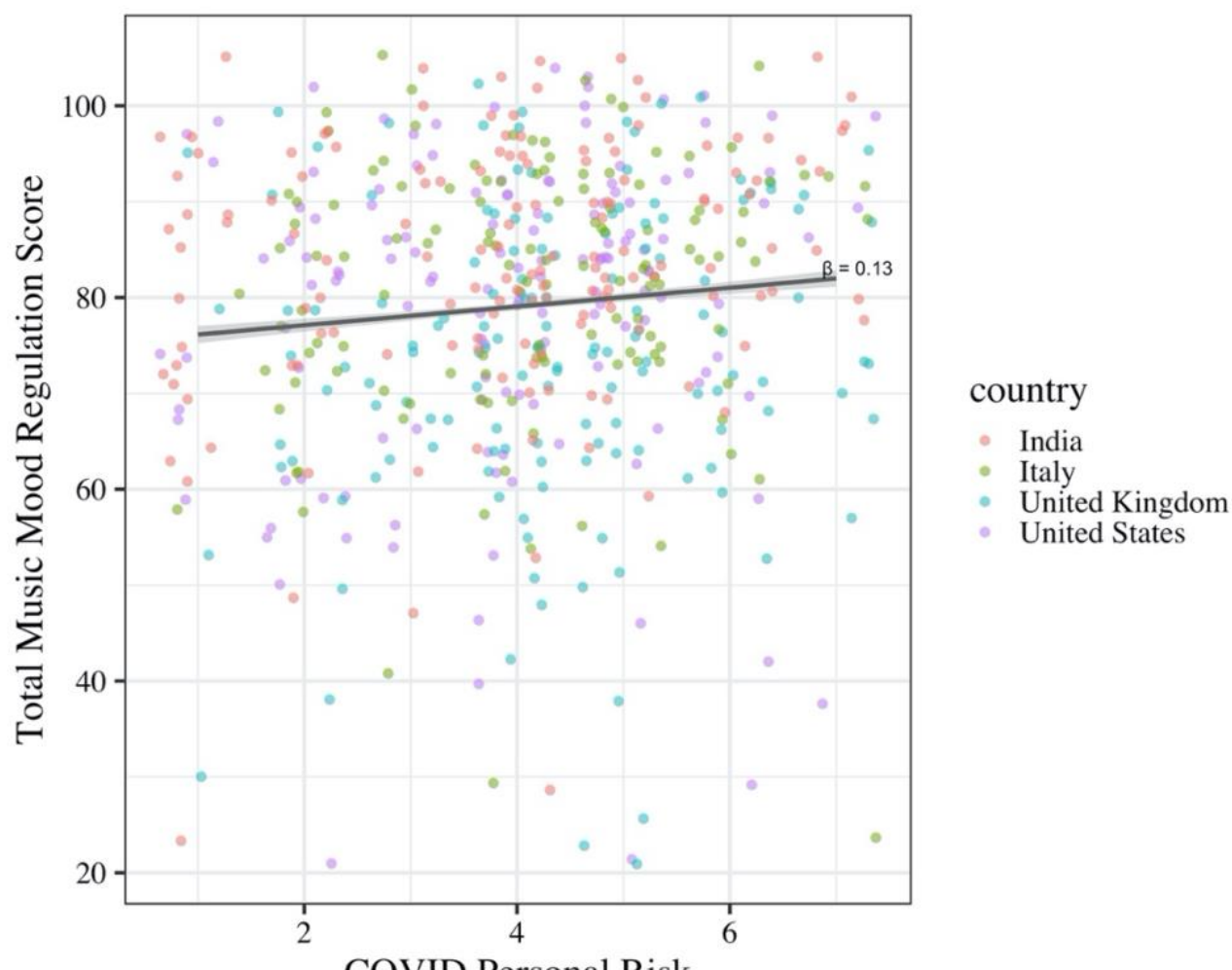

Fig 3. Association between COVID personal perceived risk and total music and mood regulation score (B-MMR Total Score) across countries.

\section{Individual differences and the function of music to regulate mood.}

The state component of the STAI significantly predicted use of the B-MMR Discharge strategy, across countries $\left(F(1,544)=17.70, \boldsymbol{\beta}=0.18, p<0.0001, \eta^{2}=0.03\right)$ (see Figure 4). State anxiety did not predict the use of any other B-MMR strategy $(p>0.05)$, nor B-MMR total $(p>0.05)$.

PHQ-9 scores significantly predicted use of the B-MMR Discharge strategy, across countries $\left(F(1,544)=51.95, \boldsymbol{\beta}=0.30, p<0.0001, \eta^{2}=0.08\right)$ (see Figure 4). PHQ-9 scores did not predict use of any other B-MMR strategy $(p>0.05)$.

The Fantasy component of empathy was positively associated with using music to regulate $\operatorname{mood}\left(F(1,541)=33.98, p<0.0001, \boldsymbol{\beta}=0.23, \eta^{2}=0.05\right)$. This was particularly true for the subscale of using music to regulate mood through Discharge $(F(1,541)=8.52, \boldsymbol{\beta}=0.12, p=$ $0.003, \eta^{2}=0.01$ ), but also entertainment, sensation, diversion, mental work, and solace. 

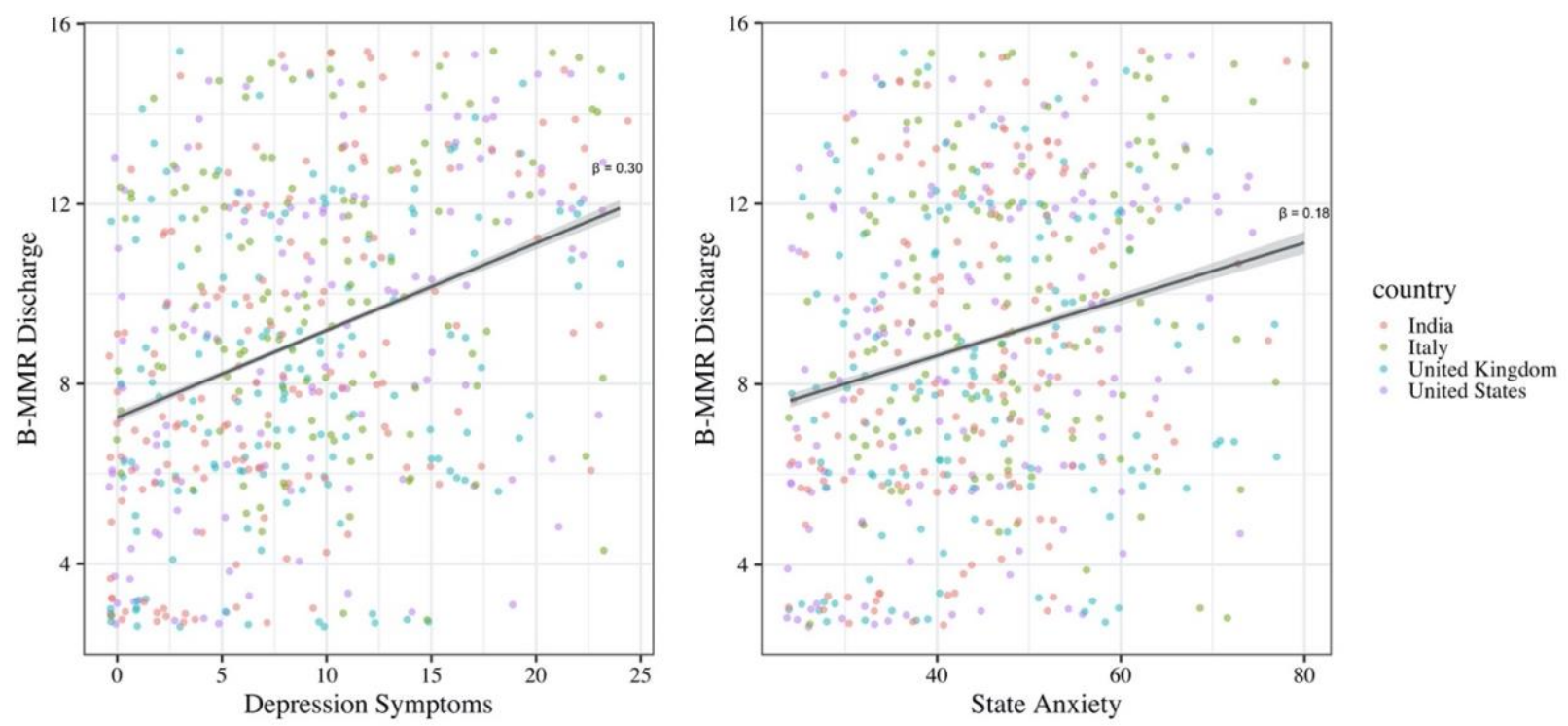

Fig 4. Association between STAI state anxiety scores and depression symptoms (PHQ score), respectively, and B-MMR Discharge strategy across countries.

Using music to regulate mood and wellbeing Mood change by country

A significant effect of country was observed on the use of music to improve mood $(F(3$, $\left.545=17.89, p<0.0001, \eta^{2}=0.09\right)$, where participants from India used music more to improve mood than did participants from Italy $(p<0.001)$, the United Kingdom $(p<0.001)$, and the United States $(p<0.001)$. Furthermore, across all countries, personal COVID-19 risk significantly predicted the use of music to improve mood $(F(1,547)=13.77, \boldsymbol{\beta}=0.16, p<0.001$, $\left.\eta^{2}=0.02\right)$. COVID -19 severity additionally predicted the use of music to improve mood across countries $\left(F(1,547)=13.90, \boldsymbol{\beta}=0.16 p<0.001, \eta^{2}=0.02\right)$.

\section{Music in Mood Regulation}

Across countries, using music to feel better was significantly associated with total scores on the B-MMR $\left(F(1,544)=348.39, \boldsymbol{\beta}=0.63, p<0.001, \eta^{2}=0.34\right)$, as well as many of the subscales, including B-MMR Discharge $\left(F(1,544)=36.98, \eta^{2}=0.26, p<0.0001, \eta^{2}=0.06\right)$, Revival $\left(F(1,544)=242.52, \boldsymbol{\beta}=0.57, p<0.0001, \eta^{2}=0.28\right)$, Diversion $(F(1,544)=282.83, \boldsymbol{\beta}=$ $\left.0.60, p<0.0001, \eta^{2}=0.30\right)$, Solace $\left(F(1,544)=259.96, \boldsymbol{\beta}=0.59, p<0.0001, \eta^{2}=0.30\right)$, Mental work $\left.(F(1,544)=195.95, \boldsymbol{\beta}=0.53), p<0.0001, \eta^{2}=0.24\right)$, Entertainment $(F(1,544)=$ 159.02, $\left.\boldsymbol{\beta}=0.50, p<0.0001, \eta^{2}=0.21\right)$, and Sensation $(F(1,544)=142.62, \boldsymbol{\beta}=0.46, p<$ $\left.0.0001, \eta^{2}=0.18\right)$ (see Figure 5). 


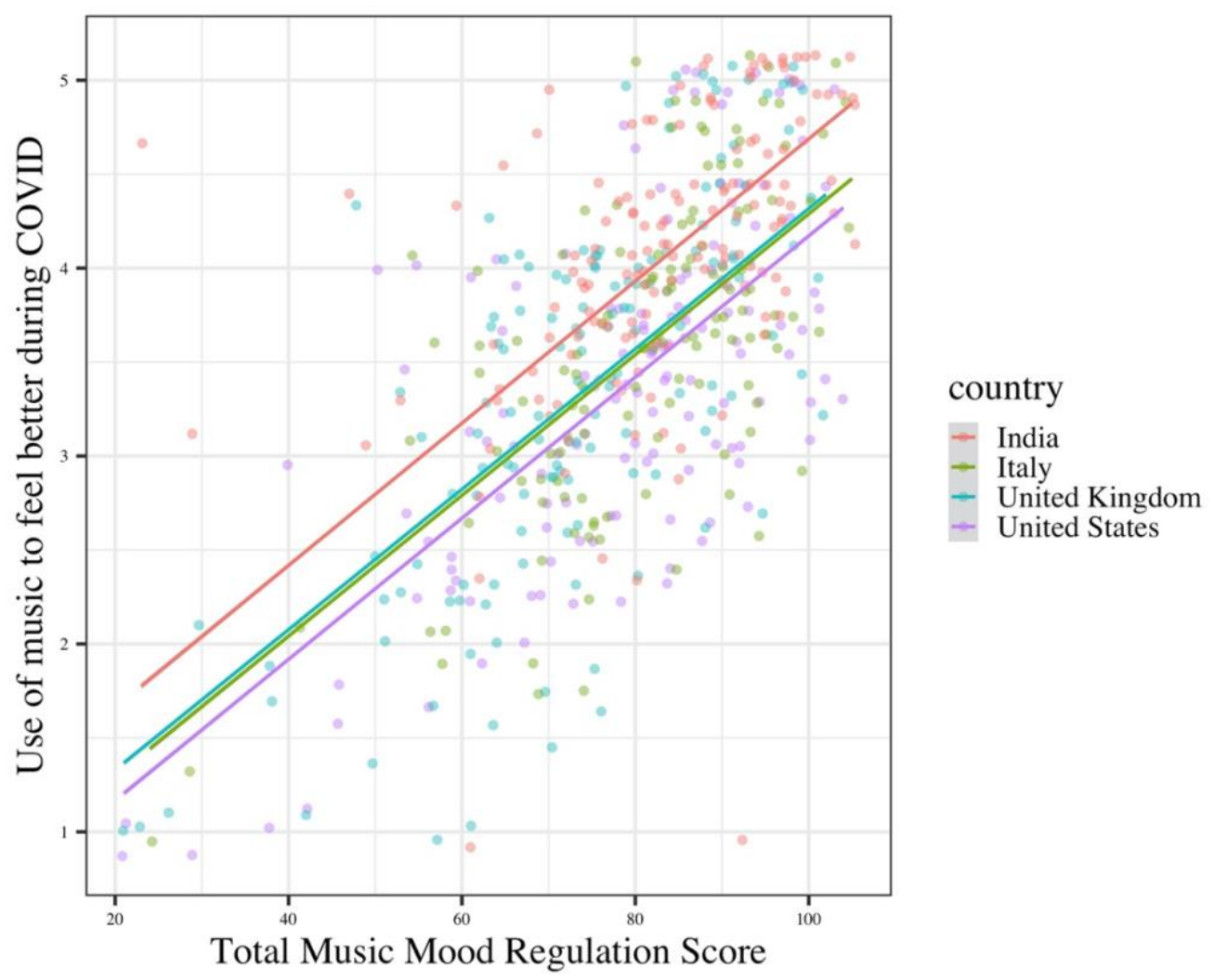

Fig 5. Association of total music and mood regulation score (B-MMR Total Score) and use of music to feel better during COVID-19 across countries.

\section{Symptoms of depression and anxiety}

PHQ-9 scores significantly predicted the use of music to feel better $(F(1,547)=8.20, \boldsymbol{\beta}$ $\left.=0.13, p<0.01, \eta^{2}=0.01\right)$. Furthermore, while no significant interaction was found between PHQ-9 scores and Discharge subscale in terms of using music to feel better $(F(1,545)=0.43, \boldsymbol{\beta}$ $\left.=-0.02, p>0.05, \eta^{2}<0.01\right)$, there was a significant interaction between these two variables on changes in mood since the start of the COVID-pandemic: that is, individuals who are more depressed and don't use music to discharge their negative feelings report feeling worse since the start of the pandemic than individuals who are depressed and use music to discharge their negative feelings $\left(F(1,546)=5.76, \boldsymbol{\beta}=0.09, p<0.05, \eta^{2}=0.01\right)$.

STAI state anxiety significantly predicted the use of music to feel better $(F(1,547)=$ $\left.3.91, \boldsymbol{\beta}=0.09, p<0.01, \eta^{2}=0.01\right)$. STAl trait anxiety scores did not predict use of music to feel better $(p>0.05)$. There was no interaction between any B-MMR strategy and state or trait anxiety scores in use of music to feel better or change of mood since the start of the pandemic (all ps $>0.05$ ).

\section{Emotion regulation}

ERQ Reappraisal predicted using music to feel better across countries $(F(1,541)=$ $30.18, p<0.001, \eta^{2}=0.05$ ) (see Figure 6), where for all four countries, higher likelihood of reappraisal was associated with greater positive mood change $(\boldsymbol{\beta}=0.27)$. There was a significant interaction between country and ERQ Suppression in using music to feel better $(F(3$, $\left.541)=3.06, \eta^{2}=0.01, p<0.05\right)$, where ERQ Suppression predicted using music to feel better 
377 in India $(\boldsymbol{\beta}=0.25)$ more than Italy $(\boldsymbol{\beta}=-0.23)$, the United States $(\boldsymbol{\beta}=-0.38)$, and the United Kingdom $(\boldsymbol{\beta}=-0.20)$ (see Figure 6).
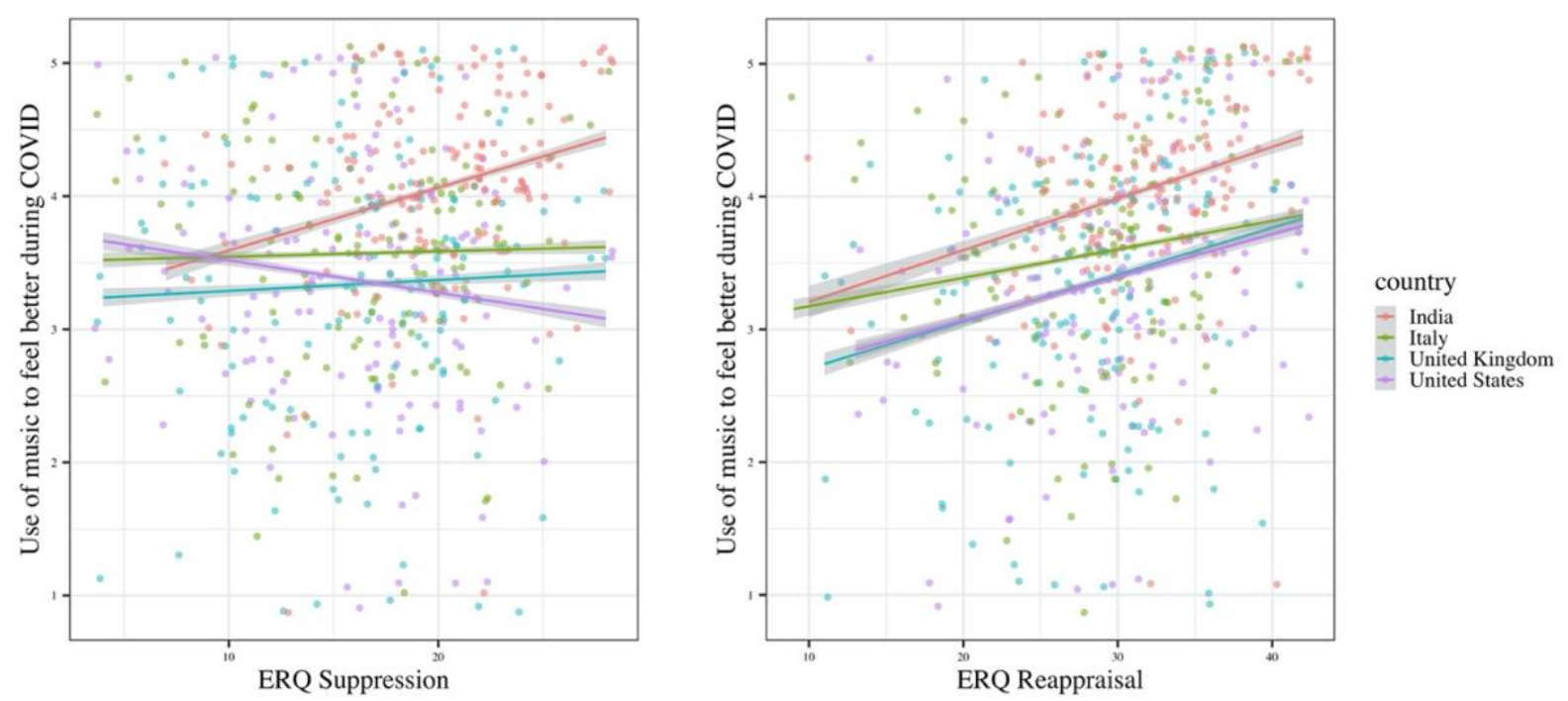

Fig 6. Association between emotion regulation strategies (ERQ Suppression and Reappraisal, respectively) and use of music to feel better during COVID-19, across countries.

\section{Empathy}

The Fantasy component of empathy was positively associated with using music to feel better during the pandemic $\left(F(1,544)=22.94, \eta^{2}=0.19, p<0.0001, \eta^{2}=0.04\right)$. Furthermore, there is a significant interaction between being personally affected by the COVID-19 pandemic and Fantasy-proneness on the Discharge component of the B-MMR $\left(F(1,542)=4.63, \eta^{2}=-\right.$ $0.09, p=<0.05, \eta^{2}=0.01$ ). That is, the relationship between Fantasy-proneness and using music to discharge negative feelings was weakened in people who felt more personally at risk of COVID-19 (see Figure 7). This relationship was not found for any other subscale of the B-MMR nor was it found with actual risk-factors associated with COVID. This suggests that people who are high in fantasy proneness are less likely to use music to discharge when feeling worse, whereas people who are low in fantasy are more likely to use music to discharge when feeling at risk of COVID. 


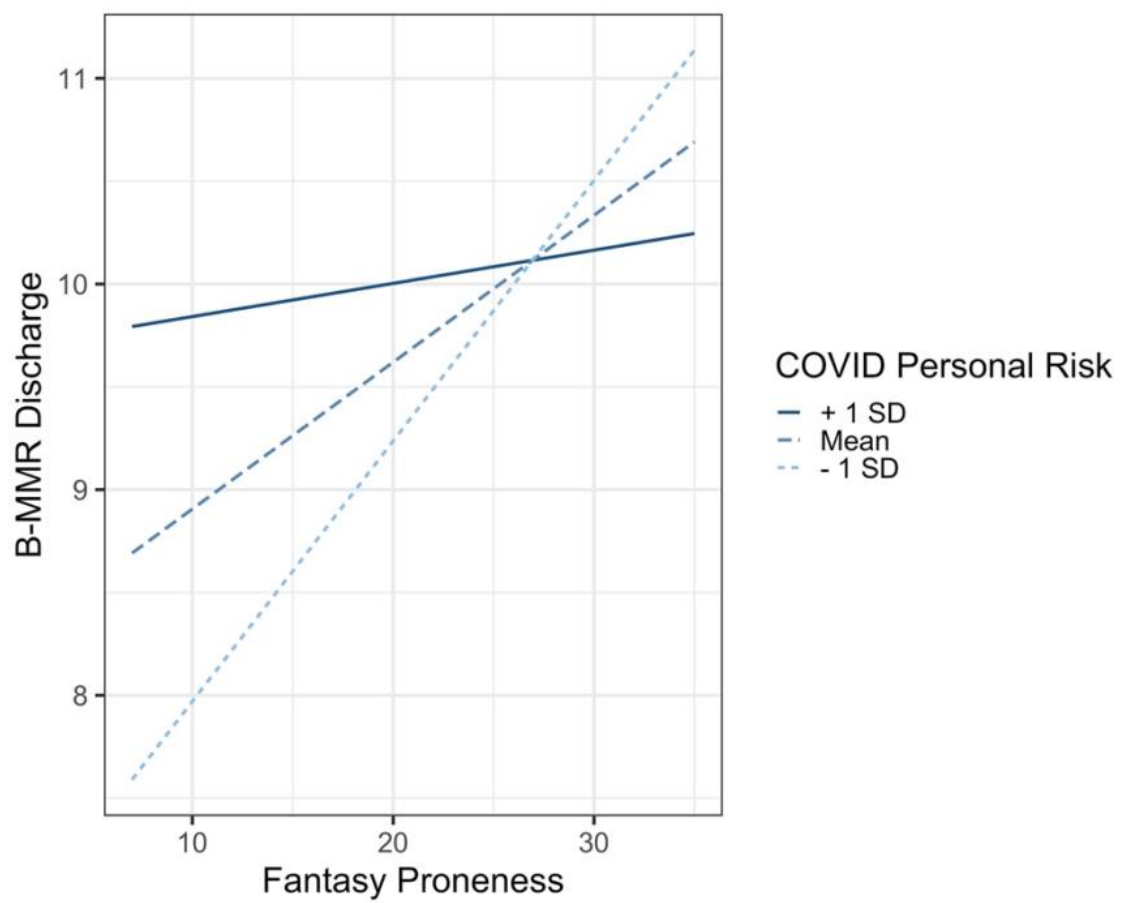

398

399

400

401

402

403

404

405

406

407

408

409

410

411

412

413

414

415

416

417

418

419

420

421

422

423

424

425

426

427

Fig 7. Mediation of personal COVID risk on the relationship between fantasy proneness (IRI fantasy factor) and Discharge strategy of B-MMR.

\section{Changes in music listening habits Audio analysis.}

No significant within-subject effects of time (pre versus post COVID) were found for any of the musical features extracted from Spotify. However, significant associations were found between the musical features of songs listened to during COVID and individual differences. COVID severity was negatively correlated with loudness $(F(1,358)=4.50, \boldsymbol{\beta}=-0.10, p=0.04$, $\left.\eta^{2}=0.01\right)$ and energy $\left(F(1,358)=5.80, \boldsymbol{\beta}=-0.12, p=0.02, \eta^{2}=0.01\right)$ and positively with acousticness $\left(F(1,358)=5.02, \boldsymbol{\beta}=0.11, p=0.03, \eta^{2}=0.01\right)$, suggesting that people who are more strongly affected by COVID are choosing to listen to music that is calmer and quieter. Acousticness and energy were also related to using music to discharge negative emotions, in the opposite direction: people who are more likely to use music as a discharge reported listening to more energetic $\left(F(1,358)=4.99, \eta^{2}=0.11, p=0.03, \eta^{2}=0.01\right)$ and less acoustic $\left(F(1,358)=4.50, \eta^{2}=-0.11, p=0.03, \eta^{2}=0.01\right)$ music during COVID. Finally, acousticness and energy were correlated with using music to feel better during the pandemic, with acousticness being positively correlated with feeling better $\left(F(1,358)=9.42, \boldsymbol{\beta}=0.16, p=0.002, \eta^{2}=0.03\right)$ and energy being marginally negatively correlated with feeling better $\left(F(1,358)=3.46, \eta^{2}=-\right.$ $\left.0.10, p=0.06, \eta^{2}=0.009\right)$. No significant interactions were found between musical features of the music, COVID-severity, and feeling better.

\section{Discussion}

In this study, we attempted to quantify the degree to which music improved mood and wellbeing during the COVID-19 pandemic. Despite varying levels of COVID-19 severity and risk, anxiety and depression across four countries, we found a significant relationship between using music 
for mood regulation and positive wellbeing. Countries with more COVID-19 cases at the time of data collection reported higher rates of state anxiety with no differences in trait anxiety nor depression. Across all four countries, people who were more personally affected by the pandemic, showed more symptoms related to depression, or were feeling more anxious were more likely to report using music to regulate mood. Using music to regulate mood also led to a positive mood change during quarantine. These results suggest that music had a salubrious impact on people during a global pandemic, transcending differences in culture, societal impact of the pandemic, and musical background.

Multiple mood regulation strategies were related to positive wellbeing, though the use of these strategies varied across countries and with individual differences. Using positive music as a diversion and as a source of renewal were strongly correlated with feeling personally at risk for COVID-19 as well as with positive mood change. This suggests that people who are feeling particularly stressed by the pandemic are turning to pleasant, calming music to feel better. This supports the recent finding that pleasant music led to decreased feelings of tiredness, sadness, fear, and worry in Italian healthcare workers in a COVID-19 hospital (Giordano et al., 2020).

On the other hand, releasing negative emotions by listening to negative-valent music was positively associated with symptoms of state anxiety and depression. Given that our analyses are correlational, it is possible that that listening to negative-valent music increases feelings of anxiety and depression. Alternatively, people who are more anxious and depressed may seek out music that conveys negative emotions for its therapeutic potential. While our results cannot directly resolve this uncertainty, there is evidence to suggest that the latter interpretation is more likely. First, we found a positive relationship between depressive symptoms and the use of music to improve mood during the pandemic. Second, we found a positive relationship between using music to discharge emotions in general and improved mood through music-listening during COVID specifically. And third, even though increased depression and anxiety were associated with feeling worse during COVID, the relationship was weaker in people who regulate their mood with music through the discharge strategy. Combined, these results suggest that people who were stressed or sad during quarantine were able to use negative-valent music to feel better, or at least, to feel less bad.

The Fantasy component of empathy was positively associated with using music to regulate mood through the discharge of negative emotions as well as with a positive mood change. Our previous work showed that Fantasy was associated with the enjoyment of sad music because it was able to elicit strong, positive emotions, suggesting that Fantasy-prone individuals are particularly suited to benefit from a discharge strategy when listening to negative-valence music (Sachs et al., 2020). Interestingly, feeling personally at-risk for contracting COVID-19 weakened the relationship between Fantasy and the discharge strategy, suggesting that the pandemic, and the stress associated with it, may attenuate the positive emotional benefits of mentally transporting into music. Whether listening to music congruent with one's negative mood is beneficial may therefore be contingent on the situation. Accordingly, in our previous study, Fantasy-proneness was positively associated with listening to sad music specifically when experiencing feelings of loneliness, but not when experiencing stress or anxiety (Sachs et al., 2020). It may be that Fantasy-prone individuals selectively use music to discharge when feeling general loneliness and sadness, but find this strategy less useful during periods of intensified anxiety due to a personal COVID risk.

A key component of this study was the examination of music and emotion regulation strategies across cultures. While participants from all countries reported using music to regulate emotions during the pandemic, participants from India reported using positive-sounding music to regulate 
mood through revival, diversion, and solace to a greater extent and ultimately reported feeling more positively. This accords with Saarikallio et al. (2020), who reported that, as compared to Finnish participants, individuals from India used music more as a mood regulation and relaxation technique. People from India also reappraise more than people from the U.S. (Mehta et al., 2017), experience less negative effects of emotional suppression (Cheung \& Park, 2010), and prefer to adjust and accommodate rather than to change or assert influence over emotionally challenging situations (Savani et al., 2011). Here, we also found that participants in India also reported using both suppression and reappraisal strategies to regulate their emotions to a greater extent than participants from other countries (see Supplemental Materials). This suggests that during such an uncontrollable and uncertain event like the COVID-19 pandemic, music may be able to improve mood by both suppressing and reappraising emotional responses, particularly for individuals living in India.

While we did not find significant within-participant differences between various musical features of songs that people chose to listen to before and during the pandemic, we did find that people who were more severely impacted by the pandemic tended to listen to music that was less loud and energetic and more acoustic. Overall, the people who listened to softer, more acoustic music also reported feeling better as a result of listening to music during the pandemic. Interestingly, people who reported using music to discharge negative feelings actually preferred music that was more energetic and less acoustic, which is consistent with the goal of using music to purge or release negative emotions.

It is important to point out that the sample collected from each country may not be representative of the country's population. Specifically, the mean age of participants skewed low, likely due to the online nature of the study. With this caveat in mind, we showed that across four different countries in three different continents, listening to music to regulate mood was a strong predictor of affective well-being during the COVID-19 pandemic. While the mechanisms by which music is able to improve mood may change across people, the fundamental result is the same. Music proves to be a powerful and salubrious tool during these unprecedented times.

\section{Acknowledgments}

The Brain and Music Program at the Brain and Creativity Institute is supported by the GRoW at Annenberg Foundation, the Los Angeles Philharmonic Association and the Van Otterloo Family Foundation. Additionally, thank you to Amita Padiyar for her contribution to this project.

\section{Author Contributions}

521

MS, JK, and AH developed the study concept. Data collection was performed by MS and SH. $\mathrm{MS}$ and $\mathrm{SH}$ performed the data analysis. MS, $\mathrm{SH}$, and $\mathrm{AH}$ drafted the manuscript, and $\mathrm{JK}$ provided critical revisions. All authors approved the final version of the manuscript for submission. 
522

523

524

525

526

527

528

529

530

531

532

533

534

535

536

537

538

539

540

541

542

543

544

545

546

547

548

549

550

551

552

553

554

555

556

557

558

559

560

561

562

563

564

565

566

567

568

569

570

571

572

\section{References}

Adler, N. E., Epel, E. S., Castellazzo, G., \& Ickovics, J. R. (2000). Relationship of subjective and objective social status with psychological and physiological functioning: Preliminary data in healthy white women. Health Psychology, 19(6), 586-592. https://doi.org/10.1037/02786133.19.6.586

Boer, D., Fischer, R., Tekman, H. G., Abubakar, A., Njenga, J., \& Zenger, M. (2012). Young people's topography of musical functions: Personal, social and cultural experiences with music across genders and six societies. International Journal of Psychology, 47(5), 355369. https://doi.org/10.1080/00207594.2012.656128

Chan, M. F., Wong, Z. Y., \& Thayala, N. V. (2011). The effectiveness of music listening in reducing depressive symptoms in adults: A systematic review. In Complementary Therapies in Medicine (Vol. 19, Issue 6, pp. 332-348). Churchill Livingstone. https://doi.org/10.1016/j.ctim.2011.08.003

Cheung, R. Y. M., \& Park, I. J. K. (2010). Anger suppression, interdependent self-construal, and depression among Asian American and European American college students. Cultural Diversity and Ethnic Minority Psychology, 16(4), 517-525. https://doi.org/10.1037/a0020655

Chin, T. C., \& Rickard, N. S. (2012). The music USE (MUSE) questionnaire: An instrument to measure engagement in music. Music Perception, 29(4), 429-446. https://doi.org/10.1525/mp.2012.29.4.429

Davis, M. H. (1980). Self Report Measures for Love and Compassion Research: Empathy INTERPERSONAL REACTIVITY INDEX (IRI). JSAS Catalog of Selected Documents in Psychology, 10(85), 3. http://fetzer.org/sites/default/files/images/stories/pdf/selfmeasures/EMPATHYInterpersonalReactivitylndex.pdf

de la Torre-Luque, A., Caparros-Gonzalez, R. A., Bastard, T., Vico, F. J., \& Buela-Casal, G. (2017). Acute stress recovery through listening to Melomics relaxing music: A randomized controlled trial. Nordic Journal of Music Therapy, 26(2), 124-141. https://doi.org/10.1080/08098131.2015.1131186

Giordano, F., Scarlata, E., Baroni, M., Gentile, E., Puntillo, F., Brienza, N., \& Gesualdo, L. (2020). Receptive music therapy to reduce stress and improve wellbeing in Italian clinical staff involved in COVID-19 pandemic: A preliminary study. Arts in Psychotherapy, 70, 101688. https://doi.org/10.1016/j.aip.2020.101688

Gross, J. J., \& John, O. P. (2003). Individual Differences in Two Emotion Regulation Processes: Implications for Affect, Relationships, and Well-Being. Journal of Personality and Social Psychology, 85(2), 348-362. https://doi.org/10.1037/0022-3514.85.2.348

Habibi, A., \& Damasio, A. (2014). Music, feelings, and the human brain. Psychomusicology: Music, Mind, and Brain, 24(1), 92-102. https://doi.org/10.1037/pmu0000033

Hanser, S., \& Mandel, S. (2010). Manage your stress and pain through music. https://doi.org/10.1093/jmt/49.4.453

Juslin, P. N., \& Laukka, P. (2004). Expression, Perception, and Induction of Musical Emotions: A Review and a Questionnaire Study of Everyday Listening. Journal of New Music Research, 33(3), 217-238. https://doi.org/10.1080/0929821042000317813

Kroenke, K., Spitzer, R. L., \& Williams, J. B. W. (2001). The PHQ-9: Validity of a brief depression severity measure. Journal of General Internal Medicine, 16(9), 606-613. https://doi.org/10.1046/j.1525-1497.2001.016009606.x

Laukka, P. (2007). Uses of music and psychological well-being among the elderly. Journal of Happiness Studies, 8(2), 215-241. https://doi.org/10.1007/s10902-006-9024-3

Loue, S., Mendez, N., \& Sajatovic, M. (2008). Preliminary evidence for the integration of music into HIV prevention for severely mentally III latinas. Journal of Immigrant and Minority 
573

574

575

576

577

578

579

580

581

582

583

584

585

586

587

588

589

590

591

592

593

594

595

596

597

598

599

600

601

602

603

604

605

606

607

608

609

610

611

612

613

614

615

616

617

618

619

620

621

622

623

Health, 10(6), 489-495. https://doi.org/10.1007/s10903-008-9142-6

Mehta, A., Young, G., Wicker, A., Barber, S., \& Suri, G. (2017). Emotion regulation choice: Differences in US and Indian populations. The International Journal of Indian Psychology, 4(2), 202-219. https://www.researchgate.net/publication/323218501

Miranda, D., \& Gaudreau, P. (2011). Music listening and emotional well-being in adolescence: A person- and variable-oriented study. Revue Europeenne de Psychologie Appliquee, 61(1), 1-11. https://doi.org/10.1016/j.erap.2010.10.002

Miranda, Dave, \& Claes, M. (2009). Music listening, coping, peer affiliation and depression in adolescence. Psychology of Music, 37(2), 215-233. https://doi.org/10.1177/0305735608097245

Palan, S., \& Schitter, C. (2018). Prolific.ac-A subject pool for online experiments. Journal of Behavioral and Experimental Finance, 17, 22-27. https://doi.org/10.1016/j.jbef.2017.12.004

Panteleeva, Y., Ceschi, G., Glowinski, D., Courvoisier, D. S., \& Grandjean, D. (2018). Music for anxiety? Meta-analysis of anxiety reduction in non-clinical samples. Psychology of Music, 46(4), 473-487. https://doi.org/10.1177/0305735617712424

R Core, T. (2020). R: A Language and Environment for Statistical Computing. R Foundation for Statistical Computing. https://www.r-project.org/

Randall, W. M., \& Rickard, N. S. (2017). PERSONAL MUSIC LISTENING: A MODEL OF EMOTIONAL OUTCOMES DEVELOPED THROUGH MOBILE EXPERIENCE SAMPLING. https://doi.org/10.1525/MP.2017.34.5.501

Saarikallio, S. (2011). Music as emotional self-regulation throughout adulthood. Psychology of Music, 39(3), 307-327. https://doi.org/10.1177/0305735610374894

Saarikallio, S. (2012). Development and validation of the Brief Music in Mood Regulation scale (B-MMR ). Music Perception, 30(1), 97-105. https://doi.org/10.1525/mp.2012.30.1.97

Saarikallio, S., Alluri, V., Maksimainen, J., \& Toiviainen, P. (2020). Emotions of music listening in Finland and in India: Comparison of an individualistic and a collectivistic culture. Psychology of Music, 0305735620917730. https://doi.org/10.1177/0305735620917730

Saarikallio, S., \& Erkkilä, J. (2007). The role of music in adolescents' mood regulation. Psychology of Music, 35(1), 88-109. https://doi.org/10.1177/0305735607068889

Saarikallio, S. H. (2012). Development and validation of the brief music in mood regulation scale (B-MMR). Music Perception, 30(1), 97-105.

https://doi.org/10.1017/CBO9781107415324.004

Sachs, M. E., Damasio, A., \& Habibi, A. (2020). Unique personality profiles predict when and why sad music is enjoyed. Psychology of Music, 030573562093266.

https://doi.org/10.1177/0305735620932660

Savani, K., Morris, M. W., Naidu, N. V. R., Kumar, S., \& Berlia, N. V. (2011). Cultural Conditioning: Understanding Interpersonal Accommodation in India and the United States in Terms of the Modal Characteristics of Interpersonal Influence Situations. Journal of Personality and Social Psychology, 100(1), 84-102. https://doi.org/10.1037/a0021083

Schäfer, T., Tipandjan, A., \& Sedlmeier, P. (2012). The functions of music and their relationship to music preference in India and Germany. International Journal of Psychology, 47(5), 370-380. https://doi.org/10.1080/00207594.2012.688133

Sloboda, J. A., O'Neill, S. A., \& Ivaldi, A. (2001). Functions of Music in Everyday Life: An Exploratory Study Using the Experience Sampling Method. Musicae Scientiae, 5(1), 9-32. https://doi.org/10.1177/102986490100500102

Spielberger, C. D. (2010). State-Trait Anxiety Inventory. In The Corsini Encyclopedia of Psychology (pp. 1-1). John Wiley \& Sons, Inc. https://doi.org/10.1002/9780470479216.corpsy0943

Suda, M., Morimoto, K., Obata, A., Koizumi, H., \& Maki, A. (2008). Emotional responses to music: Towards scientific perspectives on music therapy. NeuroReport, 19(1), 75-78. https://doi.org/10.1097/WNR.0b013e3282f3476f 
624

625

626

627

628

629

630

631

632

633

634

635

636

637

638

639

640

641

642

643

644

645

646

647

648

649

650

651

652

653

654

655
Thoma, M. V., La Marca, R., Brönnimann, R., Finkel, L., Ehlert, U., \& Nater, U. M. (2013). The Effect of Music on the Human Stress Response. PLOS ONE, 8(8), e70156. https://doi.org/10.1371/journal.pone.0070156

Thoma, M. V., Ryf, S., Mohiyeddini, C., Ehlert, U., \& Nater, U. M. (2012). Emotion regulation through listening to music in everyday situations. Cognition and Emotion, 26(3), 550-560. https://doi.org/10.1080/02699931.2011.595390

Thomson, C. J., Reece, J. E., \& Di Benedetto, M. (2014). The relationship between musicrelated mood regulation and psychopathology in young people. Musicae Scientiae, 18(2), 150-165. https://doi.org/10.1177/1029864914521422

Van den Tol, a. J. M., \& Edwards, J. (2014). Listening to sad music in adverse situations: How music selection strategies relate to self-regulatory goals, listening effects, and mood enhancement. Psychology of Music. https://doi.org/10.1177/0305735613517410

van Goethem, A., \& Sloboda, J. (2011). The functions of music for affect regulation. Musicae Scientiae, 15(2), 208-228. https://doi.org/10.1177/1029864911401174

Västfjäll, D. (2002). Influences of current mood and noise sensitivity on judgments of noise annoyance. Journal of Psychology: Interdisciplinary and Applied, 136(4), 357-370. https://doi.org/10.1080/00223980209604163

Zoteyeva, V., Forbes, D., \& Rickard, N. S. (2016). Military veterans' use of music-based emotion regulation for managing mental health issues. Psychology of Music, 44(3), 307-323. https://doi.org/10.1177/0305735614566841 
MMR_solace

MMR_mentalwork

MMR_discharge

MMR_diversion

MMR_sensation

MMR_revival

MMR_entertainment

STAI_state

phq_score

Correlation

STAI_trait

IRI_fantasy

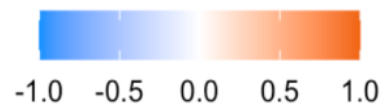

\begin{tabular}{|l|l|}
\hline $0.46 \quad 0.35$ \\
\hline
\end{tabular}

\begin{tabular}{l|l}
\hline 0.68 & 0.77
\end{tabular}
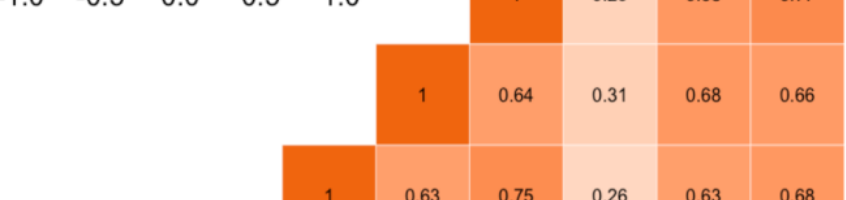


\section{COVID-19 questionnaire to assess distress related to COVID-19 pandemic}

Seven YES/NO questions were asked to assess personal COVID-19 severity. This included the following questions:

1. Do you personally know anyone that tested positive for COVID-19?

2. Do you personally know anyone that has been hospitalized due to COVID-19?

3. Do you personally know anyone that has passed away dues to COVID-19?

4. Have you made any changes to your daily lifestyle due to COVID-19?

5. Are you experiencing a financial loss due to COVID-19?

6. Is the area where you live under mandatory, "stay at home", "shelter in place" order?

7. How much are you self-isolating (from $100 \%$ to None)

The COVID-19 severity portion also included self-assessment of perceived risk and personal distress. These included the following questions:

1. In the past 4 weeks have you experienced any change in your mood? (from 1 significantly worse to 5 - significantly better)

2. In the past 4 weeks has your level of general anxiety changed? (from 1 - significantly worse to 5 - significantly better)

3. Please rate the overall level of risk to you personally that you perceive from the COVID19 pandemic (from 1 - none to 5 - extreme)

4. How much of a threat do you think COVID-19 (coronavirus) is to the world? (from 1 none to 5 - extreme)

We also asked for change in mood as a result of COVID:

5. In the past 4 weeks has your level of general anxiety changed?

6. In the past 4 weeks have you experienced any change in your mood?

\section{Additional predictors of subscales of the Brief Music in Mood Regulation Scale}

The trait component of the STAI additionally predicted the use of B-MMR strategies of Revival $\left(F(1,544)=9.57, p<0.01, \eta^{2}=0.02\right)$, and Discharge $\left(F(1,544)=31.48, p<0.0001, \eta^{2}=0.05\right)$, where higher state anxiety was negatively associated with use of Revival as a strategy ( $\boldsymbol{\beta}=-$ $0.14)$ and positively associated with use of Discharge $(\boldsymbol{\beta}=0.25)$.

ERQ Suppression scores significantly predicted use of the B-MMR Revival strategy ( $F(1$, $\left.544)=4.23, \boldsymbol{\beta}=0.09, p<0.05, \eta^{2}=0.01\right)$. ERQ Suppression did not predict use of any other $B-$ MMR strategy $(p>0.05)$. ERQ Reappraisal scores significantly predicted the use of total $B$ $\operatorname{MMR}\left(F(1,544)=42.08, \boldsymbol{\beta}=0.30, p<0.0001, \eta^{2}=0.06\right)$. ERQ Reappraisal scores also significantly predicted use of B-MMR Solace strategy $(F(1,544)=29.92, \boldsymbol{\beta}=0.23, p<0.0001$, $\left.\eta^{2}=0.05\right)$, Revival $\left(F(1,544)=53.45, \boldsymbol{\beta}=0.30, p<0.0001, \eta^{2}=0.08\right)$, Entertainment $(F(1$, $\left.544)=33.08, \boldsymbol{\beta}=0.24, p<0.0001, \eta^{2}=0.05\right)$, Sensation $(F(1,544)=42.74, \boldsymbol{\beta}=0.26, p<$ $\left.0.0001, \eta^{2}=0.06\right)$, Mental Work $\left(F(1,544)=23.24, \boldsymbol{\beta}=0.20, p<0.0001, \eta^{2}=0.04\right)$.

A significant effect of country was also observed in the reappraisal component of the $\operatorname{ERQ}\left(F(3,545)=6.04, p<0.001, \eta^{2}=0.03\right)$, where participants from India reappraised more than those from Italy $(p<0.05)$ and the United Kingdom $(p<0.001)$. A significant effect of country was also observed in the suppression component of the $\operatorname{ERQ}(F(3,545)=17 . .04, p<$ 
$\left.0.0001, \eta^{2}=0.08\right)$, where participants from India suppressed more than those from Italy $(p<$ $0.0001)$, the United Kingdom $(p<0.0001)$, and the United States $(p<0.0001)$.

\section{Additional playlist analyses}

\section{Reminiscence bump}

Music that evokes autobiographical memories has been shown to induce positive emotions (Maksimainen et al., 2018) and to mediate the relationship between emotion regulation strategy and mental health (Blais-Rochette \& Miranda, 2016). Memories evoked by music are most strongly tied to songs from an individual's "reminiscence bump" (Janata et al., 2007) defined broadly as a 10-year period in the mid-to late adolescent years (Jansari \& Parkin, 1996), characterized by rapid growth of social bonds and development of individual preferences. Engaging with feelings of nostalgia, for example through memory-evoking songs, may protect against feelings of loneliness and enforce a sense of personal meaning by reminding the listener of periods of social connectedness and identify formation (Sedikides et al., 2008; Wildschut et al., 2006). In the presence of a pandemic characterized by social isolation, it is possible that music listening habits may shift to include more memory-evoking content.

In addition to audio analyses of the songs, we were interested to know if songs listened to pre or post-COVID were more likely to fall within a participant's reminiscence bump if it was released when the participant was between the ages of 9 and 18. Reminiscence bump song percentage was calculated as the proportion of listed songs within this age period to songs outside of this age period. This was calculated for pre- and post-COVID songs separately, for Italy, the United Kingdom, and the United States. A repeated-measures ANOVA was conducted for reminiscent bump song percentage, with country as the between-subjects factor, and time as the withinsubjects factor.

No significant effect of time, country, or time $x$ country interaction was observed on reminiscence bump song percentage $(p>0.05)$.

\section{Genre}

Chi-square test of association was conducted to assess differences in musical genre from pre- to post-COVID. There was a significant association between music genre and time $\left(X^{2}\right.$ $(16, N=480)=416, p<0.0001)$. Participants listened to classical $(p<0.001)$, jazz $(p<0.0001)$, latin, rock $(p<0.001)$ music more pre-COVID-19 than post-COVID-19. Participants listened to country $(p<0.0001)$, folk $(p<0.05)$, hip-hop $(p<0.01)$, K-pop $(p<0.0001)$, musical theater $(p<$ $0.001)$, pop $(p<0.01)$, and trap $(p<0.0001)$ music post-COVID-19 than pre-COVID-19. 